TINS Opinion article:

\title{
Metastability and coherence: Extending the Communication Through Coherence hypothesis using a whole-brain computational perspective
}

\author{
Deco, Gustavo ${ }^{1}$ \& Kringelbach, Morten L. ${ }^{2,3}$ \\ ${ }^{1}$ Institució Catalana de la Recerca i Estudis Avançats (ICREA), Universitat Pompeu Fabra, Passeig Lluís Companys 23, \\ Barcelona, 08010, Spain \\ ${ }^{2}$ Department of Psychiatry, University of Oxford, Oxford OX3 7JX, UK \\ ${ }^{3}$ Center for Music in the Brain (MIB), Department of Clinical Medicine, Aarhus University, DK
}

Keywords: Communication Through Coherence, synchronization, metastability, whole-brain-modelling

\begin{abstract}
Understanding the mechanisms for communication in the brain remains one of the most challenging scientific questions. The Communication Through Coherence (CTC) hypothesis was originally proposed ten years ago, stating that two groups of neurons communicate most effectively when their excitability fluctuations are coordinated in time (i.e. coherent), and this control by cortical coherence is a fundamental brain mechanism for large-scale, distant communication. In light of new evidence from whole-brain computational modelling of multimodal neuroimaging data, we link CTC to the concept of metastability which refers to a rich exploration of the functional repertoire made possible by the underlying structural whole-brain connectivity.
\end{abstract}

\section{A fundamental principle of Communication Through Coherence}

A hallmark characteristic of neuronal activity is rhythmic oscillation in several distinct frequency bands ranging from ultraslow to ultrafast, from approximately $0.05 \mathrm{~Hz}$ to $500 \mathrm{~Hz}$, as shown by local single cell spiking activity, local field potentials, EEG, MEG, and, for the very low frequencies, fMRI. Interactions among neuronal groups depending on neuronal (zero or non-zero phase) synchronization could modulate the communication and information processing between those groups of neurons. Two groups of neurons may communicate - or exchange information - most effectively when their excitability fluctuations are coordinated in time, i.e. when they are coherent. This has been stated as the Communication Through Coherence (CTC) hypothesis, which posits that cortical coherence is a mechanism that can influence the transmission of information between neuronal populations [1-3], specifically through gamma - and beta-band $(30-90 \mathrm{~Hz})$ synchronization. Central to this hypothesis is the idea that synchronization between neuronal groups can have causal consequences for neuronal communication. Thus, oscillations are proposed to dynamically shape the computational role of different neuronal groups linked through static structural connectivity.

More specifically, the CTC principle is an example of an important, well-documented mechanism for the state dependent coupling fundamental for implementing flexible means of effective communication between different brain regions without changing the fixed underlying anatomical structure. Indeed, the CTC hypothesis posits that neuronal interactions depend on phase relations between neuronal oscillations. Phase relations that time inputs to reach their 
target at high-excitability allow an effective transmission of spikes. Consistent phase relations require the synchronization between neuronal groups. Synchronization does not necessarily have to occur at zero-phase difference, and the optimal phase relation depends on the frequency of the involved rhythms and the conduction delays between the neuronal groups. In the absence of synchronization, i.e. in the absence of a consistent phase relation (zero or non-zero), transmission will be relatively ineffective. As a consequence, the state of synchronization between different groups of neurons in different brain areas allows a flexible switch for different routes of effective communication, while maintaining the same underlying skeleton of synaptic/anatomical structural connections. Mathematical modelling has demonstrated that simply coupling oscillatory systems with a range of structural connectivity patterns will in most cases merely result in trivial fully asynchronous or synchronous states [4], both of which are inconsistent with the CTC hypothesis. For CTC mechanisms to support communication, synchronization needs to be spatially structured and temporally dynamic to link local neuronal groups into functional networks at the appropriate moments.

Here, we link the CTC hypothesis with recent progress in computational modelling of whole-brain neuroimaging data, specifically: 1) We propose that the underlying microscopic CTC-mediated interactions are reflected at the macroscopic whole-brain level of slow oscillations, as evidenced by fMRI, which serves to study the way in which the flexible effective connectivity repertoire of patterns is implemented within fixed whole-brain anatomical structural connectivity; 2) we show that a way to implement the CTC hypothesis, i.e. optimal exploration of the dynamic repertoire inherent in the brain's structural connectivity, is by maximization of the metastability. Here, we refer to metastability as a measure of the variability of the states of phase configurations as a function of time, i.e. how the synchronization between the different nodes fluctuates across time [38]. Thus, we measure the metastability as the standard deviation of the Kuramoto order parameter across time. Importantly, both of these extensions can be studied with whole-brain neuroimaging techniques such as $\mathrm{PMRI}$ and MEG of the resting state.

To showcase the importance of CTC and metastability (and vice versa), we will appeal to the important distinction between structural, functional and effective connectivity [5]. Structural connectivity corresponds to the synaptic (anatomical) connections that support dynamics. These dynamics induce statistical dependencies between nodes, known as functional connectivity. These dependencies are distinct from effective connectivity, which refers to the directed influence one node (or neuronal population) exerts over another. The key intuition that we want to convey is that coherence (a measure of functional connectivity or frequency-specific dependencies) determines effective connectivity (the directed coupling), thereby inducing a circular causality between the two measures. This is formally not unrelated to the slaving principle in synergetics [6,7]; in the sense that coherence can be regarded as an (macroscopic) order parameter that enslaves (microscopic) synaptic interactions to produce state-dependent coupling. This activity or statedependent effective connectivity underlies metastability and is a crucial aspect of self-organised dynamics in the brain.

While the main principles of the CTC hypothesis can be easily understood, the mechanistic implementation of CTC for state-dependent dynamic of effective connectivity is not trivial within the large dynamical system of the human brain. We show that CTC can exist within the dynamical system and that it is compatible and consistent with the known detailed anatomy of the human brain, provided that the brain is working at a dynamical point where the system shows maximal metastability under spontaneous conditions (i.e. not during task or sensory stimulation). Furthermore, state-ofthe-art large-scale computational modelling of the whole brain demonstrates that for the working point of the model (fitting empirical neuroimaging data from the resting state), the system exhibits maximal metastability [8]. This finding is non-trivial and can be seen as an optimal implementation of the main tenets of the CTC hypothesis, i.e. a system 
which is able to sustain a maximal number of CTC-mediated routes under rest which can be a posteriori selected effectively and flexibly by appropriate external stimulation. In other words, a broad and flexible metastable exploration of the dynamical repertoire of the brain is equivalent to the requirement that the system is able to implement a broad range of different communication routes through phase synchronisation. Within the fixed underlying anatomical structure of the human brain, this allows a flexible way of implementing a rich set of specific communication patterns or effective connectivity between different combinations of regions across the whole brain.

\section{The Neurophysiological Basis and Origin of the CTC Hypothesis}

Rhythmic synchronization in frequency bands such as theta $(4-8 \mathrm{~Hz})$, beta $(13-30 \mathrm{~Hz})$, or gamma $(30-100 \mathrm{~Hz})$ is related to alternating periods of network inhibition and excitation [9-11]. The CTC hypothesis suggests that gamma- and betarhythmic changes in excitability have important consequences for neuronal communication [1-3]. Specifically, the hypothesis states that two groups of neurons exchange information most effectively when their excitability fluctuations are coherent [12]. Conversely, if a synaptic input arrives during the inhibitory phase of the oscillation cycle, its efficacy is likely reduced (because of shunting inhibition on the neurons receiving the input). Thus the efficacy of spikes in transmitting information may be influenced by synchronization (see Figure 1a-b).

Consistent with this hypothesis, task-induced changes in synchronization or coherence have been reported at the level of individual regions during selective attention [13], working memory [14], and motor control [15]. Similarly, such taskinduced changes in synchronization have been reported between distant cortical regions during working memory [16], long-term memory encoding [17], visual attention [18], and sensorimotor integration [19] - although note that some other findings of transient induced gamma-band responses in EEG may not be linked to CTC per se but rather have arisen from miniature saccades [20]. Probably the most direct support for the CTC hypothesis was recently found in experiments, where two different groups of neurons in the lower visual area V1 and a third group in the higher visual area V4 of behaving macaque monkeys exhibited selective communication [21, 22]. Two stimuli, positioned next to each other at the same eccentricity a few degrees away from the fixation point of the monkey, were presented simultaneously. The two stimuli activated the two separate groups of neurons in V1, whereas the neuronal group in V4 was activated by either stimulus to approximately the same degree. The simultaneous presence of both stimuli induced a gamma rhythm in their respective V1 neuronal group. Crucially, only the V1 gamma induced by the stimulus, that animals were instructed to attend to, entrained the V4 gamma. The ignored stimulus induced a gamma rhythm in V1, which did not entrain the V4 gamma. In other words, the selective entrainment of V4 gamma by the attended V1 is the likely cause for the selective routing (communication) of the attended stimulus from V1 to V4, as predicted by the CTC hypothesis (see Figure 1c).

\section{Linking connectomics with CTC for whole-brain state-dependent coupling}

Much evidence for the CTC hypothesis has come from in-vivo neurophysiology in animals using e.g. multi-site spike and LFP recordings [23]. Yet such measurements are difficult to obtain in humans and limited in their spatial extent. This is not fully overcome by the usage of extended electrode grids over large cortical areas as this does not allow for access to all brain areas simultaneously. Thus, a major challenge is to relate the macroscopic level of coherence/synchronization across the whole brain with a flexible way of implementing a change of effective connectivity of an underlying hardwired anatomical structural connectivity. In this way, the findings from the CTC hypothesis can be reflected and thus tested globally, complementing in this way neurophysiological methodologies focusing on the original gamma/beta mediation of those interactions. Note, that we are not claiming that the 
coherence/synchronization mediated correlation that we observe at the whole-brain level is a mechanism per se, but rather reflecting the signature of an underlying gamma-beta-band coherence mechanism (i.e. the traditional CTC mechanism). In fact, many experimental and whole-brain computational papers shows how the slow macroscopic dynamics emerges from the underlying gamma rhythms [4, 24]. Importantly, such a whole-brain perspective would substantially expand the reach of the CTC hypothesis to the synchronization along brain-wide information routes involving many different areas. Indeed, this perspective would allow for the study of the communication routes across the whole brain in unprecedented mechanistic detail, potentially enabling understanding the changes between different states of consciousness in healthy people, as well as the breakdown of neural communication in disease [25].

The most recent advances in connectomics using the latest neuroimaging techniques (diffusion tensor imaging, DTI; functional magnetic resonance imaging, fMRI; magnetoencephalography, MEG) together with large-scale whole-brain computational modelling offer a concrete experimental/theoretical framework for reaching this crucial aim [26]. Neuroimaging data show that during cognition large-scale network dynamics exhibit complex spatiotemporal patterns. At very slow frequencies- typically less than $0.1 \mathrm{~Hz}$ - correlated activity can be seen and defines robust spatial 'networks'- even at resting, spontaneous, conditions [27, 28]. At higher frequency, patterns of oscillatory activity can change quickly, and patterns of oscillatory coherence can be seen in multiple frequency bands during task execution. As many cognitive processes involve distributed activity across the brain, understanding the dynamics at the whole-brain scale may provide key insights into the nature of cognition.

While neurophysiological methods in other animals have been instrumental in forming our understanding of synchronization thus far, evidence is now emerging that human neuroimaging techniques such as fMRI and MEG can also be used to investigate the state of synchronization at different time scales across the whole brain reflecting the underlying CTC-mediated routes. Under resting state conditions, the grand average functional connectivity (FC) of a resting brain summarizes the well-structured spatial correlations between different brain areas. Furthermore, wholebrain models explicitly linking spontaneous local neuronal dynamics with the structural connectivity (SC) of the brain (as e.g. mapped using probabilistic tractography of DTI data) are able to explain the emergence of the spatial resting correlations (see Box 1).

Spontaneous resting activity is not, however, structured only spatially but also exhibits common temporal structure, which is characterized by transitions, switching between a few discrete FC states across time. Most studies have only investigated the grand average FC, i.e. across the whole available time window, which is defined as the matrix of correlations of hemodynamic BOLD signals between all brain regions over the whole time window of acquisition (Figure 2A). Yet to characterize the time-dependent structure of the resting fluctuations requires an FC dynamics (FCD) matrix [29] which can be constructed from smaller sliding windows of activity (see Figure 2B-C) or simply based on the phases of the signal (phase-based FCD) in the following way: 1) each full-length BOLD signal is first band-pass filtered within the narrowband of $0.04-0.07 \mathrm{~Hz}$, and the time-varying phase $\varphi_{k}(t)$ of each narrow-band signal $k$ is computed using the Hilbert transform; 2) the time-varying phases of all areas, $\varphi_{k}(t)$, define a brain coherence state; 3 ) this is a symmetric matrix where the $(\mathrm{t} 1, \mathrm{t} 2)$ entry is defined by the overlap or phase relation (i.e. cosine of the phase difference) between the corresponding brain states $\varphi_{k}(\mathrm{t} 1)$ and $\varphi_{k}(\mathrm{t} 2)$. Epochs of stable brain state configurations are reflected around the FCD diagonal in blocks of overlapping elevated inter- $\varphi_{k}(\mathrm{t})$. Computing the phase FCD statistics simply requires collecting the upper triangular elements of the matrices (over all participants or sessions) and generating their distributions, which can then be compared (Figure 2D). 
The output of whole-brain computational models (fitted to the empirical data) can be submitted to the same phase-based FCD analysis. Comparing the simulated and empirical distributions requires comparing the means of the KolmogorovSmirnov distance between them. The Kolmogorov-Smirnov distance quantifies the maximal difference between the cumulative distribution function, the phase based FCD analyses characterize the richness/multiplicity of brain states across time, which can be interpreted as the available CTC-like routes for state-dependent coupling. Note that these CTC-like routes are possibly reflections, at the level of slow/macroscopic BOLD signal, i.e. $0.05 \mathrm{~Hz}$ filtered data, of the underlying gamma-beta band mediated CTC mechanisms.

Simply positing a circular causality between functional and effective connectivity does not speak to a specific link between metastability and CTC mechanisms. There are many mechanisms that induce state-dependent coupling in the brain; for example neuromodulatory and mean field effects. For example, it is well known that increases in mean activity levels increases effective connectivity [30]. The particular form of state-dependent coupling assumed by the original CTC hypothesis depends upon precise phase relationships at gamma/beta frequencies. This is interesting because coupled phase oscillator (e.g., Kuramoto) models of neuronal dynamics can be cast explicitly in terms of the phase entrainment and effective connectivity among the phases of an oscillation [24, 31]. Technically, this dependency is encoded in something called a phase interaction function. The maintenance of a particular communication channel through coherence suggests a persistent phase relationship. Similarly, the number or repertoire of such coupling dynamics corresponds to the number or variability of phase relationships. This is important because the variability of the Kuramoto order parameter (that reflects the phase relationships) can then be used to quantify the number of realised CTC-like channels. In the following, we will use proxies for this variability to illustrate the implicit repertoire afforded to the brain. Crucially, the variability of the Kuramoto order parameter (and its proxies from dynamic functional connectivity) measure metastability [32].

Thus, computing the phase-based FCD of empirical and simulated resting data can help characterize the CTC-mediated routes. It is also possible to extend this analysis to task-based activity. The flexible selection of different CTC- mediated routes under different task-based conditions requires resting activity to have access to many possible CTC-mediated routes, i.e. exhibit a broad exploration of the underlying dynamical repertoire. In this key scenario, a "gentle" change triggered by external stimuli or a task can easily bias communication to different CTC-mediated routes. In terms of dynamical systems, this scenario implies that the CTC hypothesis would require the resting brain to exhibit maximal metastability, refining and providing evidence in favour of the synergetics hypothesis of Haken [6] (later further explored by Tognoli and Kelso [33]).

\section{Whole-Brain Computational Modelling, Metastability and CTC-mediated Routes}

While phase-based FCD is a powerful tool for measuring the spatiotemporal unfolding of functional connectivity, a mechanistic understanding of brain function and underlying computational mechanisms, such as those posited by the original CTC hypothesis can be aided by whole-brain computational modelling. Such models are key to understanding the relationships between maximal metastability and the flexibility between different routes available to CTC and CTClike mechanisms at the global level of neuroimaging. A particularly powerful computational approach is use of the normal form of a Hopf bifurcation at the level of each brain region. Research has shown the usefulness, richness and 
generality of this type of model for combining the best of oscillatory and asynchronous models and thus describing the temporal dynamics of EEG at the local node level $[34,35]$.

The possible extension of the original CTC hypothesis to investigate CTC-like state-dependent coupling has been explored using whole-brain computational modelling with the Hopf bifurcation, linking the underlying brain architecture (SC, structural connectivity) with the dynamics of neuronal activity (Figure 3). In whole-brain computational models, network topology is derived from DTI, where coupling strength is typically assumed to be equal for all connections and can be scaled with a global scaling factor [36]. The whole-brain computational model is constrained by the SC, and fitted to empirical neuroimaging data, whether coming from fMRI [36, 37] or MEG [38]. Figure 3A-D shows how the Hopf model $[34,35]$ fits empirical BOLD resting state data as a function of changing the global coupling strength. In particular, the best fitting of the dynamical spatiotemporal measurements (FC, FCD and maximal metastability) supports the availability of CTC-like routes (the variability of FCD is shown in Figure 3A; the FCD fitting in Figure 3B and metastability in Figure 3C). As can be seen from these figures, the richness of the exploration of the dynamical repertoire during the resting state (i.e. the multiplicity of synchronized brain states) is strongly constraining to a limited working region. The grand-averaged FC (Figure 3D) also fits well, but is less sensitive to the optimum working region in terms of coupling strength. Indeed, large regions of the coupling parameters are consistent with the empirical data. Instead, the figure shows that the best fitting of the spatiotemporal characteristics of the empirical resting fMRI data can be found as the minimum of the Kolmogorov-Smirnov distance between empirical and simulated FCD (Fig. 3B). This optimum corresponds to a working region where the metastability is maximal (Figure 3C), and where the temporal variability of the FCD is also maximal (Figure 2A). These two measurements characterize the richness of the exploration of the dynamical repertoire in terms of multiplicity of visited synchronized brain states, and shows that the largest repertoire of effective patterns (or routes) are found when the model is maximally metastable.

Notably, the model achieves the observed high metastability and high variability/multiplicity of synchronized brain states only for a particular scaling of coupling strength in the very particular underlying SC structure derived from fMRI of the healthy human brain [26]. Simply coupling oscillatory systems with different connectivity structures will in most cases result merely in trivial fully asynchronous or synchronous states [4], which are both inconsistent with the availability of sufficient routes for the CTC hypothesis. Instead, there may well be something important about the particular non-random, non-regular, but more specific pattern of the underlying anatomical SC of the brain which is important for implementing and sustaining a rich repertoire of effective connectivity.

Indeed, as shown in Figure 3E, damage to selective parts of this structure significantly impairs the ability of the system to sustain this dynamic repertoire of potential CTC-like routes. As can be seen, if the connections of a group of nodes in the SC are damaged, this results in a decrease of the level of synchronization (mean FCD) and variability (std FCD), i.e. a deterioration of the repertoire (Figure 3E). In particular, the results are strongly dependent on which exact nodes of the $\mathrm{SC}$ are damaged. Figure 3E shows the results obtained for the normal intact SC, and for the damaging of the 12 top nodes with regard to either 1) being non-rich club members (i.e. nodes with lowest degree), 2) being rich-club members (highest degree) [39], and 3) being members of a binding club (highest reduction of entropy) [26]. The largest deterioration comes from pruning the nodes fulfilling central structural (RC) or dynamical (BC) roles. 


\section{Concluding remarks}

In this opinion article we have explored the potential of the original CTC hypothesis to cast light on whole-brain mechanisms for communication made possible by coherence both at the microscopic and mesoscopic level. In particular we have explored the links between metastability, an important signature of a healthy brain, and that of the CTC mechanisms of state-dependent coherence. We have shown that the original CTC hypothesis is a well-documented important mechanism for a fast rhythm of cognition and we are proposing that these mechanisms are reflected on a much slower, even ultraslow timescale that is equally important for studying cognition. We are proposing that a flexible modulation of effective connectivity require maximal metastability to allow for a full exploration of the rich functional repertoire made possible by normal structural brain connectivity.

Still, it is important to consider the functional asymmetry in brain networks. Current methods do not take this into account at the level of undirected (symmetric) measures such as coherence, and often overlook the fact that the brain is hierarchically organised, i.e. ascending connections are very different from descending connections. This is an outstanding challenge for descriptions of brain dynamics in terms of globally coupled maps.

Here, we have shown that emerging evidence from topological analysis of structural connectivity in the human brain combined with whole-brain computational modelling supports the idea that the CTC hypothesis supports a flexible communication between areas. Specifically, the evidence suggests that the healthy brain is maximally metastable, which is required for the optimal exploration of the effective dynamical repertoire of patterns. If the underlying structural connectivity is damaged, as is found in many brain disorders, a much more limited repertoire of CTC routes is available, with potentially severe consequences. For example, it has been shown that the anterior insula and cingulate cortex are part of the common neurobiological substrate for mental illness across six diverse diagnostic groups (schizophrenia, bipolar disorder, depression, addiction, obsessive-compulsive disorder, and anxiety) based on a metaanalysis of grey matter loss in 193 neuroimaging studies of 15892 individuals [40]. There is evidence from whole-brain computational models that removing these and other regions results in a particularly strong reduction of the underlying repertoire as compared to the healthy brain [41]. This strongly suggests that CTC mechanisms might be impaired in neuropsychiatric disorders, perhaps differentially depending on the underlying changes in structural connectivity. As such this reinforces the potential use of CTC hypothesis together with whole-brain computational modelling for understanding the underlying mechanisms of brain disorders [25].

\section{Box 1: Whole-Brain Models}

At the global level, the brain can be described by a large-scale network of local neural networks -or nodes, linked by long-range connections. The global dynamics of the whole-brain network is determined by the intrinsic dynamics of the nodes, i.e. the dynamics of a node in absence of all couplings, and the network couplings, which allow the communication between the nodes of the network. The local spontaneous dynamics of a single node can be modelled as attractors of a network of spiking neurons coupled through AMPA, NMDA and GABA receptor synaptic dynamics [42] and can be captured by neural mass models. Indeed, the emergent collective macroscopic behaviour of brain models has been shown to be only weakly dependent on the details of individual neuron behaviour [43]. There are two types of reduced models, namely those that have used a dynamic mean field model derived from a proper reduction of a detailed spiking neuron model, which itself is asynchronous by definition [44, 45], and those including a-priori oscillatory units $[37,38,46]$. It is possible to combine both, asynchronous and oscillatory scenarios for obtaining a more realistic description of the characteristic of the measured signals. For example, a neural mass description based on the most 
general form that expresses both noisy asynchronous dynamics and oscillations, namely a normal form of a Hopf bifurcation [34, 35], has been recently proposed [8].

The structure of the physical couplings is specified by the underlying anatomical skeleton, the so-called structural connectivity (SC) matrix. In humans, this type of anatomical information, i.e. the SC matrix, can be estimated from Diffusion Tensor Imaging (DTI), where white matter fibers connecting distant neural populations can be modeled via tractography algorithms. On the other hand, the global characterization of the whole-brain dynamics is usually described by the functional connectivity matrix (FC) which expresses the statistical dependence between brain regions of neurophysiological signals as recorded with indirect measures such as functional MRI and PET, or with direct measures of neuronal activity such as MEG and EEG [47, 48]. The dynamical entrainment and correlations between different local brain region dynamics are crucially shaped by the underlying anatomical structural connectivity [37, 4953]. Thus, whole-brain models explicitly link the underlying anatomical SC matrix, shaping the interplay between the local dynamics of each node, with the FC matrix, characterizing the global dynamics of the brain. Indeed, whole-brain models can provide a mechanistic explanation of the origin of resting state dynamics (i.e. no stimulation, no task). Recently, several studies have successfully done so for both resting state MRI [42, 54] and resting state MEG [24]. A whole-brain model has also been used to model important features of sleep [55]. Furthermore, whole-brain models are not only able to describe grand averaged spatial FC correlations, but also spatiotemporal aspects of the dynamics as specified by the FC dynamics, the FCD. The FCD captures the spatiotemporal organization of FC by representing the similarities between FC(t) matrices calculated in a certain window of time (usually $60 \mathrm{sec}$ ) located at different times $t$ [29].

\section{Acknowledgements}

We want to thank Pascal Fries and the two anonymous reviewers for their helpful suggestions. GD is supported by the ERC Advanced Grant: DYSTRUCTURE (n. 295129) and by the Spanish Research Project PSI2013-42091. MLK is supported by the ERC Consolidator Grant: CAREGIVING (n. 615539). 


\section{References}

1 Fries, P. (2005) A mechanism for cognitive dynamics: neuronal communication through neuronal coherence. Trends in cognitive sciences $9,474-480$

2 Fries, P. (2009) Neuronal gamma-band synchronization as a fundamental process in cortical computation. Annual review of neuroscience 32, 209-224

3 Fries, P. (2015) Rhythms for Cognition: Communication through Coherence. Neuron 88, 220-235

4 Cabral, J., et al. (2011) Role of local network oscillations in resting-state functional connectivity. NeuroImage 57, 130-139

5 Friston, K.J., et al. (2003) Dynamic causal modelling. NeuroImage 19, 1273-1302

6 Haken, H. (1975) Cooperative phenomena in systems far from thermal equilibrium and in nonphysical systems. Reviews of Modern Physics 47, 67-121

7 Haken, H. (1988) Information and Self-Organization. A macroscopic approach to Complex Systems. Springer

8 Kringelbach, M.L., et al. (2015) The rediscovery of slowness: exploring the timing of cognition. TICS 19, 616-628

9 Buzsaki, G., et al. (2003) Hippocampal network patterns of activity in the mouse. Neuroscience 116, 201211

10 Hasenstaub, A., et al. (2005) Inhibitory postsynaptic potentials carry synchronized frequency information in active cortical networks. Neuron 47, 423-435

11 Kopell, N., et al. (2000) Gamma rhythms and beta rhythms have different synchronization properties. Proceedings of the National Academy of Sciences of the United States of America 97, 1867-1872

12 Buehlmann, A. and Deco, G. (2010) Optimal information transfer in the cortex through synchronization. PLoS computational biology 6, e1000934

13 Womelsdorf, T., et al. (2006) Gamma-band synchronization in visual cortex predicts speed of change detection. Nature 439, 733-736

14 Howard, M.W., et al. (2003) Gamma oscillations correlate with working memory load in humans. Cereb Cortex 13, 1369-1374

15 Ball, T., et al. (2008) Movement related activity in the high gamma range of the human EEG. NeuroImage 41, 302-310

16 Jones, M.W. and Wilson, M.A. (2005) Theta rhythms coordinate hippocampal-prefrontal interactions in a spatial memory task. PLoS biology 3, e402

17 Fell, J., et al. (2001) Human memory formation is accompanied by rhinal-hippocampal coupling and decoupling. Nature neuroscience 4, 1259-1264

18 Gregoriou, G.G., et al. (2009) High-frequency, long-range coupling between prefrontal and visual cortex during attention. Science $324,1207-1210$

19 Roelfsema, P.R., et al. (1997) Visuomotor integration is associated with zero time-lag synchronization among cortical areas. Nature 385, 157-161 
20 Yuval-Greenberg, S., et al. (2008) Transient induced gamma-band response in EEG as a manifestation of miniature saccades. Neuron 58, 429-441

21 Bosman, C.A., et al. (2012) Attentional stimulus selection through selective synchronization between monkey visual areas. Neuron $75,875-888$

22 Grothe, I., et al. (2012) Switching neuronal inputs by differential modulations of gamma-band phasecoherence. The Journal of neuroscience : the official journal of the Society for Neuroscience 32, 1617216180

23 Schoffelen, J.M., et al. (2005) Neuronal coherence as a mechanism of effective corticospinal interaction. Science 308, 111-113

24 Cabral, J., et al. (2014) Exploring mechanisms of spontaneous functional connectivity in MEG: How delayed network interactions lead to structured amplitude envelopes of band-pass filtered oscillations. Neuroimage 90, 423-435

25 Deco, G. and Kringelbach, M.L. (2014) Great Expectations: Using Whole-Brain Computational Connectomics for Understanding Neuropsychiatric Disorders. Neuron 84, 892-905

26 Deco, G., et al. (2015) Rethinking segregation and integration: contributions of whole-brain modelling Nat Rev Neurosci 16, 430-439

27 Biswal, B., et al. (1995) Functional connectivity in the motor cortex of resting human brain using echoplanar MRI. Magnetic resonance in medicine : official journal of the Society of Magnetic Resonance in Medicine / Society of Magnetic Resonance in Medicine 34, 537-541

28 Smith, S.M., et al. (2012) Temporally-independent functional modes of spontaneous brain activity. Proceedings of the National Academy of Sciences of the United States of America 109, 3131-3136

29 Hansen, E.C., et al. (2015) Functional connectivity dynamics: modeling the switching behavior of the resting state. NeuroImage 105, 525-535

30 Chawla, D., et al. (1999) The relationship between synchronization among neuronal populations and their mean activity levels. Neural computation 11, 1389-1411

31 Kuramoto, Y. (1984) Chemical Oscillations, Waves, and Turbulence. Springer-Verlag, Berlin

32 Wildie, M. and Shanahan, M. (2012) Metastability and chimera states in modular delay and pulse-coupled oscillator networks. Chaos 22, 043131

33 Tognoli, E. and Kelso, J.A. (2014) The metastable brain. Neuron 81, 35-48

34 Freyer, F., et al. (2011) Biophysical mechanisms of multistability in resting-state cortical rhythms. The Journal of neuroscience : the official journal of the Society for Neuroscience 31, 6353-6361

35 Freyer, F., et al. (2012) A canonical model of multistability and scale-invariance in biological systems. PLoS Comput Biol 8, e1002634

36 Deco, G., et al. (2009) Key role of coupling, delay, and noise in resting brain fluctuations. Proceedings of the National Academy of Sciences of the United States of America 106, 10302-10307

37 Ghosh, A., et al. (2008) Noise during rest enables the exploration of the brain's dynamic repertoire. PLoS computational biology 4, e1000196 
38 Cabral, J., et al. (2014) Exploring the network dynamics underlying brain activity during rest. Prog Neurobiol 114, 102-131

39 van den Heuvel, M.P. and Sporns, O. (2011) Rich-club organization of the human connectome. The Journal of neuroscience : the official journal of the Society for Neuroscience 31, 15775-15786

40 Goodkind, M., et al. (2015) Identification of a Common Neurobiological Substrate for Mental Illness. JAMA psychiatry $72,305-315$

41 Honey, C.J. and Sporns, O. (2008) Dynamical consequences of lesions in cortical networks. Human brain mapping 29, 802-809

42 Deco, G. and Jirsa, V.K. (2012) Ongoing cortical activity at rest: criticality, multistability, and ghost attractors. The Journal of neuroscience : the official journal of the Society for Neuroscience 32, 3366-3375

43 Breakspear, M. and Jirsa, V. (2007) Neuronal Dynamics and Brain Connectivity. In Handbook of brain connectivity (Jirsa, V.K. and McIntosh, A.R., eds), pp. 3-64, Springer

44 Deco, G., et al. (2013) Resting-state functional connectivity emerges from structurally and dynamically shaped slow linear fluctuations. The Journal of neuroscience : the official journal of the Society for Neuroscience 33, 11239-11252

45 Deco, G. and Kringelbach, M.L. (2014) Great Expectations: Using Whole-Brain Computational Connectomics for Understanding Neuropsychiatric Disorders. Neuron 84, 892-905

46 Deco, G., et al. (2009) Key role of coupling, delay, and noise in resting brain fluctuations. Proc Natl Acad Sci USA

47 Bassett, D.S., et al. (2011) Dynamic reconfiguration of human brain networks during learning. Proceedings of the National Academy of Sciences of the United States of America 108, 7641-7646

48 Stam, C.J., et al. (2009) Graph theoretical analysis of magnetoencephalographic functional connectivity in Alzheimer's disease. Brain : a journal of neurology 132, 213-224

49 Honey, C.J., et al. (2009) Predicting human resting-state functional connectivity from structural connectivity. Proceedings of the National Academy of Sciences of the United States of America 106, 20352040

50 Deco, G., et al. (2014) Identification of optimal structural connectivity using functional connectivity and neural modeling. The Journal of neuroscience : the official journal of the Society for Neuroscience 34, 79107916

51 Deco, G., et al. (2014) How local excitation-inhibition ratio impacts the whole brain dynamics. The Journal of neuroscience : the official journal of the Society for Neuroscience 34, 7886-7898

52 Deco, G., et al. (2013) Resting brains never rest: computational insights into potential cognitive architectures. Trends in neurosciences 36, 268-274

53 Deco, G., et al. (2011) Emerging concepts for the dynamical organization of resting-state activity in the brain. Nature reviews. Neuroscience 12, 43-56

54 Honey, C.J., et al. (2007) Network structure of cerebral cortex shapes functional connectivity on multiple time scales. Proceedings of the National Academy of Sciences of the United States of America 104, 1024010245 
55 Deco, G., et al. (2014) Modeling resting-state functional networks when the cortex falls asleep: local and global changes. Cerebral cortex 24, 3180-3194

56 Tzourio-Mazoyer, N., et al. (2002) Automated anatomical labeling of activations in SPM using a macroscopic anatomical parcellation of the MNI MRI single-subject brain. NeuroImage 15, 273-289 
A

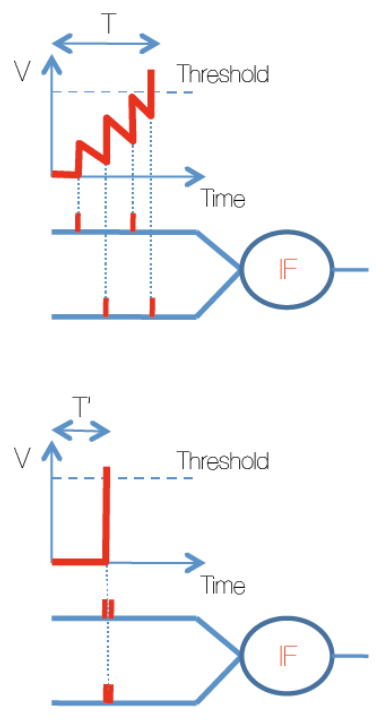

B

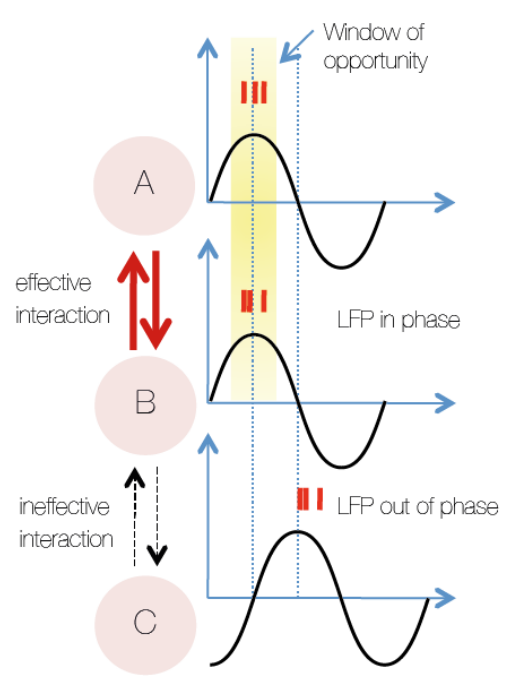

C

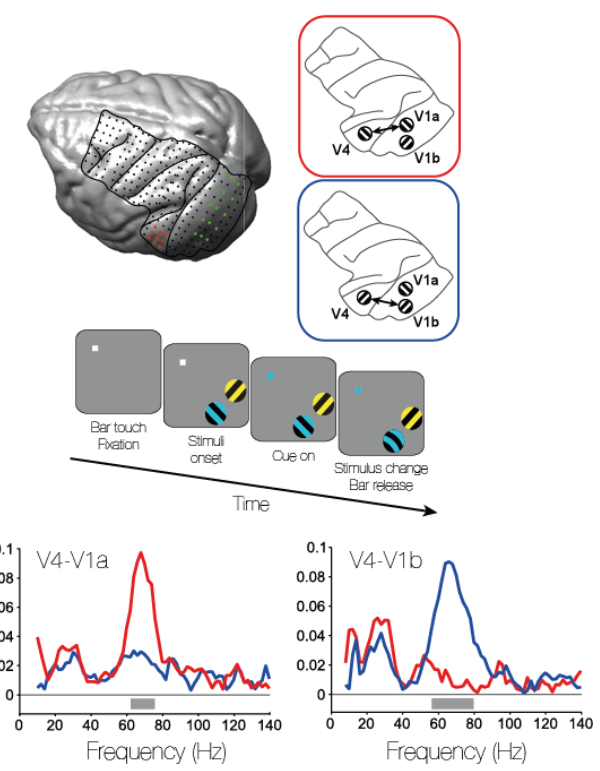

Figure 1: Principles by which synchronicity and oscillations can influence neural processing. (a) Synchronous spikes from different sources (below) may speed neuronal responses compared to asynchronous spikes (above). $V$ is the membrane potential of an integrate-and-fire neuron (IF). The time taken to reach the threshold for firing is T'vs T. (b) The Communication Through Coherence (CTC) theory suggests that effective connections in a network can be shaped through phase relations $[1,2]$. The neurons inside the pools $A, B$ and $C$ are rhythmically synchronized as indicated by the sinusoidal background LFP and the spikes (vertical red bars) around the peaks. Pools $A$ and $B$ are in phase and therefore the interchange of spikes is more effective, and more information is transmitted. On the other hand, pools $B$ and $C$ are in anti-phase and therefore fewer spikes are produced in the receiving population, and less information is transmitted. (c) Bosman et al. (2012) main experimental result: Simultaneous recordings in two different groups of neurons in VI (VI a and V1b) corresponding respectively to two different stimuli, and in a third group in V4 of behaving monkeys. The simultaneous presence of both stimuli induced a gamma rhythm in their respective V1 neuronal group. Only the attended VI gamma managed to entrain the V4 gamma, whereas the ignored stimulus induced a gamma rhythm in V1, which did not entrain the V4 gamma. 
A

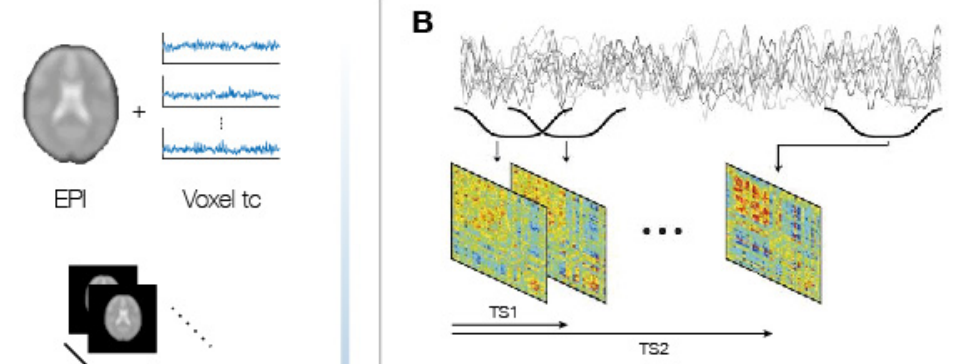

EPI timeseries

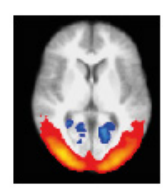

rs-MRI

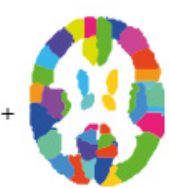

AAL

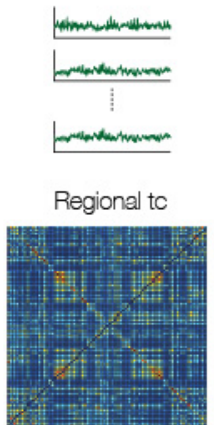

FC matrix
C

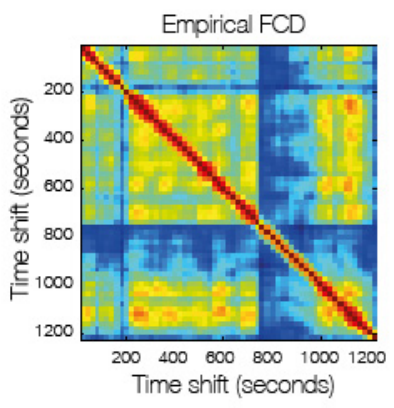

D

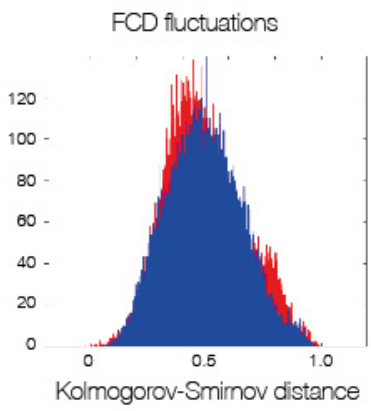

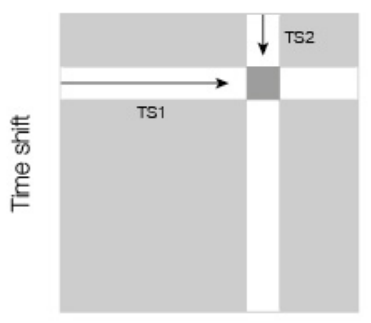

Time shift

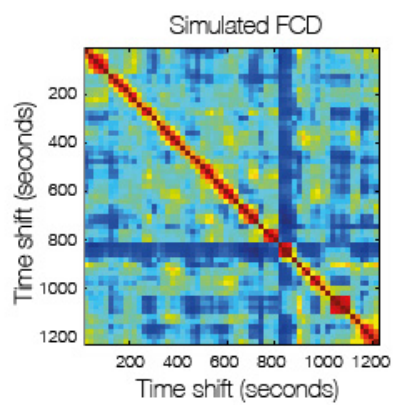

E
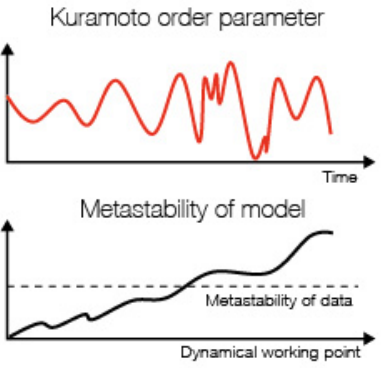

Figure 2. Measuring grand average functional connectivity (FC) and FC dynamics (FCD). A) The grand average functional connectivity (FC) can be obtained from functional neuroimaging measures of brain activity; using, for example, echo planar imaging (EPI) scans that sample the blood-oxygen-level dependent (BOLD) signal timecourse (tc) in each voxel. Combining this with a parcellation scheme (such as the AAL [56]) can recreate the regional timecourses for each of the regions in the parcellation. The grand average FC matrix is typically created from correlating the regional timecourses. B) The FCD captures the spatiotemporal organization of FC by representing the similarities between FC(t) matrices, e.g. calculated in over a short sliding windows of time located at different times, for example TS1 and TS2. C) The FCD is thus a symmetric matrix where an entry (ts1, ts2) entry is defined by the Pearson correlation between $F C(t s 1)$ and $F C(t s 2)$. Epochs of stable $F C(t)$ configurations are thus reflected around the FCD diagonal in blocks of elevated inter-FC(t) correlations in empirical (left) andsimulated (right) data. (D) For comparing the FCD statistics, the upper triangular elements of the matrices are collected over all participants and sessions. The simulated and empirical distributions can then be compared by calculating the Kolmogorov-Smirnov distance between them, because this quantifies the maximal difference between the cumulative distribution functions of the two samples. (E) The metastability can be measured as the SD of the Kuramoto order parameter across time, which provides a measure the global level of synchronization of the oscillating signals. 
A

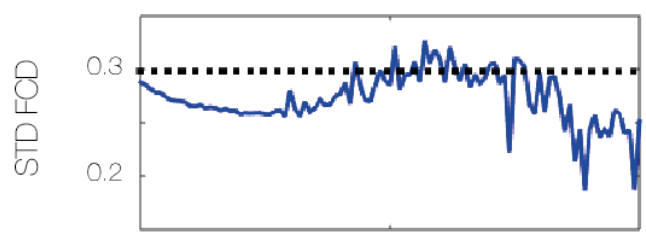

B

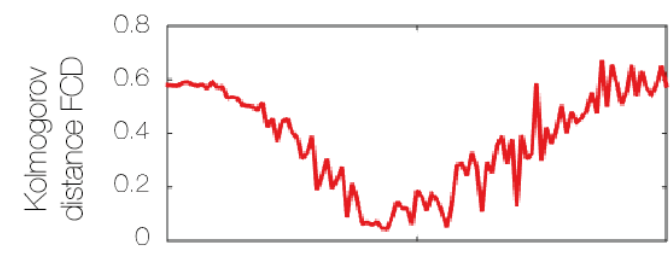

C

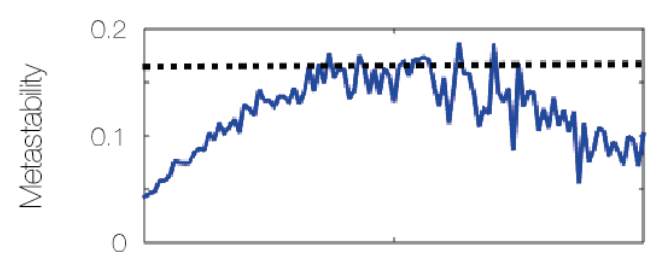

D

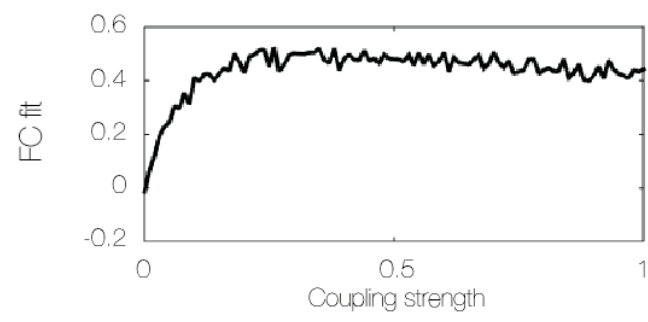

E

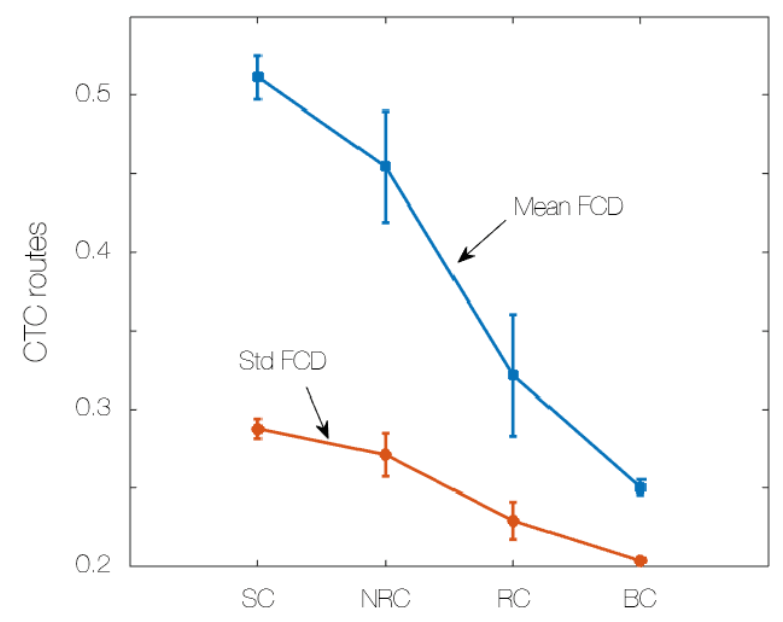

Figure 3.Investigating the optimality of CTC routes using whole-brain modelling. The figure shows the fit of a wholebrain computational Hopf model to spontaneous BOLD data from healthy participants as a function of the global coupling strength, i.e. the global synaptic efficacy scaling the underlying DTI based structural connectivity matrix used in the model. A) The CTC routes are shown as the variability (standard deviation) of the phase based FCD of the model (blue line) as function of coupling strength, compared the standard deviation of the FCD for the empirical data (dashed line). B) The best fit between the model and empirical data was found by calculating the Kolmogorov-Smirnov distance between the empirical and simulated FCD as a function of the coupling strength (red line, minimum at around 0.45). C) Similar findings are shown for the level of metastability as a function of the coupling strength (blue line), where the dashed line is the empirical metastability of the empirical data. D) Fitting of the grand-averaged FC. E) Using the optimal model parameters the available dynamic CTC routes can found for various underlying brain architectures e.g. the full normal brain model (SC) and to models with various degrees of damage to regional connectivity. As can been the mean FCD and std FCD decrease monotonically as the SC has removed the 12 low degree regions (non-rich club, NRC), followed by SC without 12 top rich club regions (RC) [39] which have been proposed to form a structural backbone of the human brain. Finally, the most damage to CTC routes was found in SC without the 12 top binding club nodes (BC) [26]. 


\section{Glossary}

Attractor

A set to which a dynamical system evolves after a long enough time. Points that get close to the attractor remain close, even under small perturbations.

\section{Bifurcation}

An abrupt qualitative change in the system's dynamics when one or more parameter pass through critical values, for instance the loss of stability and appearance of sustained oscillations.

\section{Connectome}

The complete description of the structural connections between elements of a nervous system.

\section{Criticality}

At the brink of a bifurcation, the system displays certain characteristic dynamic features, of which most are related to enhanced fluctuations.

\section{Critical slowing down}

The phenomenon that the return time of a disturbance back to equilibrium increases close to a bifurcation.

\section{Dynamical systems}

An area of applied mathematics that describes the behaviour of complex (possibly chaotic) dynamical systems as described by differential or difference equations.

\section{Diffusion tensor imaging (DTI)}

An MRI technique that takes advantage of the restricted diffusion of water through myelinated nerve fibres in the brain to infer the anatomical connectivity between brain regions.

\section{Duty cycle}

The percentage of one period in which a signal is active.

\section{Edges}

In a brain graph, an edge between nodes (regions or neurons) indicates that the nodes are anatomically or functionally connected.

\section{Functional connectivity}

Statistical association - for example, significant correlations — between neurophysiological measurements recorded from anatomically distinct neurons or regions at several time points.

\section{Graph theory}


A branch of mathematics that deals with the formal description and analysis of graphs. A graph is defined simply as a set of nodes (vertices) linked by connections (edges), and may be directed or undirected. When describing a real-world system, a graph provides an abstract representation of the system's elements and their interactions.

\section{Hopf bifurcation}

In nonlinear dynamics, a Hopf bifurcation is a local bifurcation in which an initially stable fixed point of a dynamical system loses its stability in an oscillatory fashion.

\section{Kuramoto order parameter}

A measure of the level of synchrony between oscillators in a network.

\section{Mean-Field model}

The mean-field approximation consists of replacing the temporally averaged discharge rate of a cell with an equivalent momentary activity of a neural population (ensemble average) that corresponds to the assumption of ergodicity. According to this approximation, we characterize each cell assembly by means of its activity population rate.

\section{Metastability}

In dynamical systems refers to a state which falls outside the natural equilibrium state of the system but persists for an extended period of time. Here metastability can be simply measured as the standard deviation of the Kuramoto order parameter $\left(R(t)=\left|\sum_{k=1}^{n} e^{i \varphi_{k}(t)}\right| / n\right)$ across time (Figure 2E)

\section{Magnetoencephalography (MEG)}

A method of measuring brain activity by detecting minute perturbations in the extracranial magnetic field that are generated by the electrical activity of neuronal populations. 